Cahiers
Recherche
surles Droits

Fondamentaux

Cahiers de la recherche sur les droits

fondamentaux

6 | 2008

Pouvoirs exceptionnels et droits fondamentaux

\title{
La norme et l'exception. Réflexions sur les rapports du droit avec la réalité
}

Jean-Christophe Le Coustumer

\section{OpenEdition}

Édition électronique

URL : https://journals.openedition.org/crdf/6802

DOI : $10.4000 /$ crdf.6802

ISSN : 2264-1246

Éditeur

Presses universitaires de Caen

Édition imprimée

Date de publication : 31 décembre 2008

Pagination : 19-28

ISBN : 978-2-84133-259-5

ISSN : $1634-8842$

Référence électronique

Jean-Christophe Le Coustumer, «La norme et l'exception. Réflexions sur les rapports du droit avec la réalité », Cahiers de la recherche sur les droits fondamentaux [En ligne], 6 | 2008, mis en ligne le 11

décembre 2020, consulté le 14 novembre 2022. URL : http://journals.openedition.org/crdf/6802 ; DOI : https://doi.org/10.4000/crdf.6802 


\title{
La norme et l'exception. Réflexions sur les rapports du droit avec la réalité
}

\author{
Jean-Christophe LE COUSTUMER \\ Professeur à I'Université de Rouen
}

\begin{abstract}
I. Définir la norme par l'exception ou l'exception par la norme. La querelle sur le gardien de la Constitution
II. Normes et jugement, norme de jugement. Juger de la réalité par le droit

III. Juger de l'action sur la réalité par la norme. L'exemple de l'égalité et de ses effets
\end{abstract}

Norme et exception ont chacune deux significations qui peuvent être distinguées mais qui se tiennent entre elles et renvoient les unes aux autres. Dans son acception la plus ordinaire, la norme signifie ce qui est normal, ce qu'il est normal, c'est-à-dire admis, justifié, de faire, de voir, de dire, dans une certaine société à un moment donné. Mais la norme, c'est aussi ce qu'il est obligatoire de faire dans le même contexte.

L'exception, pour sa part, est, par opposition radicale à la norme, ce qui justement ne peut pas être compris dans le champ d'une norme. Mais l'exceptionnel c'est aussi ce qui, à l'intérieur du champ de la norme, déroge à la règle, au sens matériel, posée par la norme.

Par exemple, s'il existe une norme qui veut que l'on ôte son chapeau devant une dame, alors sera exceptionnel le comportement qui consistera à ne pas ôter ledit couvrechef dans la circonstance en question. Mais cet acte exceptionnel peut tout à fait être récupéré par la norme s'il existe dans le système normatif de la politesse une règle particulière qui prévoit des cas dans lesquels il est autorisé de déroger de façon limitée à la règle présentée comme générale. Mais cet évènement peut aussi être exceptionnel car il est proprement hors de toute normativité, mais s'assume ou se reconnait comme tel.

Si le caractère de l'exceptionnel semble profondément attaché, par son lien d'ailleurs avec une situation normative, à l'éphémère, au momentané, il implique aussi un rapport à la règle, le rapport d'un certain fait à une règle. Cette idée, que l'on retrouve dans l'expression bien connue "c'est l'exception qui confirme la règle", montre qu'il y a donc une fondation réciproque des notions de règle et d'exception. En un sens, là où n'existe pas de règle, de système de règles, il ne peut y avoir d'exception, car il n'existe rien alors à quoi comparer la chose que l'on présente comme exceptionnelle. Pour qu'une exception soit, il faut donc qu'elle s'oppose à une règle, qui elle-même permet de donner signification d'exception à un acte quelconque. L'exception entretient donc un rapport au système de règles lui-même ainsi qu'à une règle en particulier.

S’interroger sur les liens entre la norme et l'exception, entre la normativité et l'exceptionnel sous un angle spécifiquement juridique, revient donc assez largement à réfléchir aux rapports complexes qu'entretiennent le droit (système de règles) et les faits, ou, autrement dit, aux rapports qui existent entre le droit et ce que l'on appelle, avec toutes les ambiguïtés évidentes, la réalité. Le droit est un domaine et une discipline évidemment propice à ce genre de réflexion sur les rapports entre un énoncé normatif et une réalité à laquelle il s'adresse, sur laquelle il veut agir. Mais s'attaquer à ce problème du rapprochement entre la grammaire juridique et la réalité des cas de façon générale et exhaustive est bien sûr une vaine tentative tant les angles d'approche peuvent être nombreux et l'on risque alors de se perdre, quelque part entre droit et réalité. 
Dans les pages qui suivent, je proposerai donc quelques réflexions sur le thème de la norme et de l'exception perçu surtout à travers le lien entre le fait et le droit.

J'envisagerai tout d'abord les rapports entre norme et exception à travers la célèbre querelle du gardien de la Constitution qui opposa Carl Schmitt et Hans Kelsen au début du $\mathrm{XX}^{\mathrm{e}}$ siècle en ce qu'elle illustre très clairement l'opposition de deux conceptions assez radicalement inconciliables en la matière, puis, dérivant vers une réflexion sur ce que c'est que, et comment l'on peut, juger de la réalité par le droit nous verrons comment le réalisme décisionniste tente de faire se rejoindre norme et réalité. Enfin, à travers l'exemple du principe d'égalité, droit fondamental par excellence, et de ses exceptions, nous terminerons par une interrogation sur les difficultés qui apparaissent quand l'on s'interroge sur les effets des normes juridiques sur la réalité.

\section{Définir la norme par l'exception ou l'exception par la norme. La querelle sur le gardien de la Constitution}

Le rapport du normatif à l'exceptionnel est parfaitement illustré, en ce qui concerne les limites entre le fait et le droit, le langage sur les faits et le langage du droit, dans la fameuse querelle qui opposa Kelsen et Schmitt autour $\mathrm{du}$ «gardien de la Constitution». Pour Kelsen, le gardien de la norme supérieure ne peut être qu'une cour constitutionnelle, sur le modèle de celle qu'il a participé à mettre en place en Autriche en 1920. Pour Schmitt en revanche, le gardien de la Constitution, c'est le Président du Reich.

Tout ce débat sur la protection de la Constitution prend évidemment racine dans les deux conceptions radicalement différentes de la notion, et la fonction de la Constitution elle-même chez Kelsen et Schmitt.

Pour Kelsen, on le sait, la Constitution est une norme, une norme qui fonde et clôt le système juridique. C'est la norme qui permet à toutes les autres normes d'être valides, celle à laquelle on remonte de degré en degré dans la hiérarchie des normes. Kelsen a donc une conception que l'on peut dire normative et dynamique, c'est-à-dire largement formelle, de la Constitution.

Carl Schmitt défend pour sa part une conception plus existentielle de la Constitution, et s'il en distingue un certain nombre de définitions, c'est une conception absolue qui retient particulièrement son attention. Pour Schmitt, la Constitution est d'abord un tout unitaire qui caractérise, identifie la décision d'une autorité souveraine, le pouvoir constituant, dont Schmitt donne la célèbre définition selon laquelle «le pouvoir constituant, c'est la volonté dont le pouvoir ou l'autorité sont en mesure de prendre la décision globale, concrète sur le genre et la forme de l'existence politique propre ${ }^{1}$.

Schmitt ne refuse pas par principe l'idée de norme, ou de normativité ${ }^{2}$, ce qu'il contesterait dans la représentation kelsénienne c'est l'idée que tout l'ordre juridique est fondé sur une norme « dont la justesse serait la raison de sa validité ». Pour Schmitt, il y a dans toute norme un moment arbitraire, "exceptionnel », indifférent au contenu de cette norme, celui de la décision. Pour lui, « tout ordre repose sur une décision, même l'ordre juridique repose sur une décision et non sur une norme $»^{3}$.

Schmitt ne rejette donc pas la notion de norme, mais l'idée que l'ordre lui-même est fondé uniquement sur une norme dont l'existence, c'est-à-dire la validité en termes kelséniens, reposerait sur une idée de la raison (la «norme fondamentale » de Kelsen). Pour Schmitt, la Constitution repose sur la décision politique d'une entité sur son identité, qui précède toute norme et qui exprime donc beaucoup plus que la simple rationalisation d'une idée ${ }^{4}$. Elle est essentiellement basée sur l'expression d'une volonté globale politique en ce sens-là et « le mot de volonté définit la nature essentiellement existentielle de ce fondement de la validité, par opposition à toute dépendance envers une justesse normative ou abstraite $»^{5}$.

La pensée de Schmitt est une pensée concrète de l'ordre, ou de l'ordre concret, un ordre qui est fondé sur une décision souveraine, une décision qui met fin au chaos et vient instaurer l'ordre politique, l'État, conçu comme une médiation entre le droit naturel et le droit positif. L'État, défini, fondé constitutionnellement, incarne donc la sortie de l'exception permanente, du désordre ${ }^{6}$.

Mais simultanément Schmitt définit le souverain comme celui qui peut décider de l'état d'exception et on aperçoit que le rapport à l'exceptionnel marque le passage de l'extérieur à l'intérieur d'un ordre, conçu d'ailleurs politiquement ou juridiquement. Giorgio Agamben décrit très bien ce phénomène quand il écrit que «l'objectif [de Schmitt] est d'inscrire l'état d'exception dans un contexte juridique. Schmitt sait parfaitement que l'état d'exception, en tant qu'il met en œuvre une "suspension de l'ordre juridique dans son ensemble", semble se "soustraire à toute considération de droit"; mais il s'agit précisément pour lui d'assurer une relation quelle qu'elle soit entre l'état d'exception et l'ordre juridique [...]. Cette articulation est paradoxale dès lors que ce qui doit être inscrit à l'intérieur du droit se révèle lui être essentiellement extérieur puisqu'il ne correspond à rien moins qu'à la suspension de l'ordre juridique lui-même. [...] Le souverain,

1. C. Schmitt, Théorie de la Constitution, Paris, PUF, 1994, p. 211.

2. Lucien Jaume parle même de «normativisme schmittien », qui serait détectable dans une «norme de l'absence de norme » incarné par l'arbitraire subjectif de Schmitt. Cf. L. Jaume, «Carl Schmitt, la politique de l'inimitié », Historia constitucional (revista electronica), ${ }^{\circ}$ 5, 2004, http://hc.rediris.es/05/ articulos/pdf/11.pdf.

3. C. Schmitt, Théologie politique, Paris, Gallimard, 1988, p. 20.

4. Cf. J.-F. Kervégan, «La critique schmittienne du normativisme kelsénien », in Le droit, le politique: autour de Max Weber, Hans Kelsen, Carl Schmitt, C.M. Herrera (dir.), Paris, L'Harmattan, 1995, p. $228 s q$

5. C. Schmitt, Théorie de la Constitution, p. 212

6. «La décision souveraine est commencement absolu, et le commencement n'est rien d'autre qu'une décision souveraine. Elle jaillit d'un néant normatif et d'un désordre concret» (C. Schmitt, Les trois types de pensée juridique, Paris, PUF, 1998, p. 83). 
qui peut décider de l'état d'exception, garantit son ancrage dans l'ordre juridique. [...] Être à l'extérieur et cependant appartenir: telle est la structure topologique de l'état d'exception ${ }^{7}$.

Et en effet c'est là toute la question : le gardien de la Constitution doit-il pouvoir être exclu du fonctionnement constitutionnel, doit-il pouvoir s'exclure du fonctionnement constitutionnel ou au contraire doit-il être une autorité dont le comportement est lui aussi juridiquement réglé ? Pour Kelsen, on le sait, c'est une cour constitutionnelle qui est l'instance chargée de faire respecter ce qu'est la Constitution, c'est-à-dire une norme, par l'usage de la norme elle-même à travers la technique du contrôle de constitutionnalité. Pour Schmitt, un contrôle juridictionnel des normes constitutionnelles est inconcevable, et ceci car il développe une conception particulière de l'acte de juger, qu'il couple avec sa conception de ce qu'exprime le texte constitutionnel.

Comme le montre remarquablement en effet Stanley Paulson, un élément central de cette querelle réside dans la conception différente qu'ont Kelsen et Schmitt du jugement judiciaire. Schmitt développe une conception assez stricte du jugement judiciaire, en voyant dans celui-ci une opération de subsomption et de déduction. Or, pour que cette déduction (de la norme au cas) puisse avoir lieu, le texte de référence doit être suffisamment clair et précis. Pour Schmitt, le texte constitutionnel ne permet pas, par sa nature même, cette déduction, qui est à l'œuvre dans la technique du contrôle de constitutionnalité. Comme l'analyse Stanley Paulson, « dès lors que le soi-disant jugement constitutionnel ne peut pas être compris dans le modèle de la subsomption - ne peut pas, autrement dit, être compris comme une déduction - il ne peut pas être considéré comme un jugement ${ }^{8}$.

Kelsen a alors beau jeu de critiquer cette conception un peu trop «classique» de Schmitt mais sa critique va porter surtout sur l'idée qu'il n'y aurait aucun « fait » dont on pourrait juger dans un jugement de constitutionnalité. Pour Kelsen en effet, « totalement erronée est la prétention selon laquelle une décision sur la constitutionnalité d'une disposition législative ne représente pas une telle subsomption par rapport à un fait matériel $»^{9}$. Le jugement porte bien sur un fait, qui est constitué par la production de l'acte législatif. Et le jugement de constitutionnalité permet de dire si ce fait respecte bien la Constitution, formellement et matériellement. Garder la Constitution prend donc chez Kelsen le tour d'une réflexion sur le rapport entre un fait (l'acte d'édiction d'une norme inférieure) et le droit en vigueur, alors que chez Schmitt, cette réflexion est tout entière concentrée sur une conception de la Constitution qui rend difficile cette représentation de ce qu'est un jugement de constitutionnalité.
Mais assez paradoxalement, les deux conceptions opposées des auteurs dans la querelle sur le jugement de constitutionnalité semblent se rejoindre dans l'idée du caractère politique de tout jugement, si l'on entend par politique la reconnaissance d'un moment indéterminé, qui ne peut être logiquement attaché à une norme, dans chaque jugement, dans chaque décision. Ni l'un ni l'autre ne s'opposeraient sur l'indétermination, ou sur une certaine indétermination de la signification du sens, de la valeur du texte à appliquer ou à interpréter.

Comment donc alors juger de l'exception? Si l'exception est réellement exceptionnelle, alors elle ne peut entrer, presque par définition, dans le champ de la norme et alors il n'est en effet pas de syllogisme possible.

À moins que la norme n'ait prévu le cas, mais alors est-il exceptionnel ? Juger de l'exception, du réellement exceptionnel et non du seulement dérogatoire, peut parầtre là encore impossible. Comment le mode de mise en œuvre du devoir-être pourrait-il saisir ce pur «être», ce pur fait qu'est une situation exceptionnelle?

Cette problématique de l'exception et de la norme nous ramène finalement une fois encore à une réflexion sur le rapport entre le fait et le droit, mais maintenant cette réflexion se décale vers le rapport entre la norme comprise comme un commandement assorti d'une sanction (au sens large) et la norme au sens social du terme.

\section{Normes etjugement, norme de jugement. Juger de la réalité par le droit}

Le terme "norme» est en effet remarquablement ambigu. Il peut signifier ce qui est la norme, au sens de la normalité empiriquement constatée, comme les règles de la politesse ou de l'habillement (c'est la régularité). Il peut signifier ce que doit être le comportement et il est alors commandement et un étalon de comparaison entre la réalité et ce commandement formulé. Si les deux sens peuvent être distingués, ils renvoient l'un à l'autre. La norme au premier sens est souvent le produit de la norme au second sens, et la norme au second sens ne peut aller complètement contre la norme au premier sens.

Une norme, dans les deux sens du terme, est une norme du jugement. Mais le fait, la réalité, doit pouvoir être comparé, confronté à la norme, à son énoncé. Si le fait est réellement exceptionnel, s'il est anormal, comment peut-il entrer dans le champ de la norme objectivement valide a priori ? Comment peut-on émettre un jugement de conformité ou de non-conformité à une norme dont on peut déterminer la signification a priori, si cette signification est censée régler les cas de son application?

C'est là avoir bien sûr une conception de la norme assez restrictive que de concevoir qu'elle a a priori, c'està-dire indépendamment de l'expérience, déjà prévu la

7. G. Agamben, «L'état d'exception », disponible sur http://www.libertysecurity.org/elise/article46.html. François Saint-Bonnet est critique de la conception de Schmitt sur l'état d'exception. Selon lui, le projet de Schmitt «est moins de rendre compte de la spécificité de l'état d'exception que de proposer, en mettant à mal l'idéologie libérale, une théorie du droit radicalement alternative sous-tendue par une philosophie politique » (F. Saint-Bonnet, L'état d'exception, Paris, PUF, 2001, p. 310).

8. S. Paulson, «Arguments conceptuels de Schmitt à l'encontre du contrôle de constitutionnalité et réponses de Kelsen », in Le droit, le politique..., p. 248.

9. H. Kelsen, Wer soll der hüter der Verfassung sein, cité par S. Paulson, «Arguments conceptuels de Schmitt... », p. 253. 
façon dont devrons être réglés les cas. Cette conception permet évidemment d'éviter la problématique des lacunes dans le droit, mais elle présente une conception de la norme juridique qui sépare radicalement celle-ci de la réalité, sur le mode de la séparation radicale entre l'être et le devoir-être. Le chemin va de la norme à la réalité, de celleci ne pouvant être inférée aucune norme, aucune valeur.

Norme sociale et norme juridique restent alors radicalement séparées, ce qui ne veut pas dire qu'elles ne sont pas en rapport l'une avec l'autre, mais leur mode de validité reste radicalement, par principe, différent. L'exception ne peut donc se comprendre que dans la norme, comme un cas qui entre quand même dans le champ de la norme mais nécessite que la règle décide elle-même comme étant l'exception, c'est-à-dire juste ce qui déroge à l'application des critères généraux de la norme.

En un certain sens, l'exception est donc (juridiquement) créée par la norme, et le traitement normatif qu’elle subit est justifié par le fait qu'il existe une norme. C'est ce que montre remarquablement Guillaume Tusseau dans un article consacré à l'urgence en droit constitutionnel, en affirmant que les normes consacrées au traitement de l'urgence permettent de modifier selon le droit tout à la fois la répartition des compétences entre les auteurs de normes, ainsi que les pouvoirs, les compétences elles-mêmes.

Et notamment en matière de droits fondamentaux, l'urgence « justifie la mise en cause des normes constitutionnelles relatives à ce que l'on considère comme les "libertés fondamentales" ou les "droits fondamentaux". Alors que leur respect s'impose avec la plus grande vigueur sous l'empire de circonstances normales, l'urgence s'accommode de leur mise à l'écart. Selon leur rédaction, soit les constitutions énumèrent les droits fondamentaux qui peuvent être suspendus en cas d'urgence, soit ceux qui ne peuvent pas l'être. Quelle que soit la manière dont sont rédigés les textes, l'urgence conduit à un accroissement du champ de normes productibles par les acteurs antérieurement habilités ${ }^{10}$.

Cette conception, qui connaît évidemment des nuances et des variantes, s'oppose à une autre conception de la norme juridique, qui voit dans celle-ci, toujours au sens kelsénien, non un acte de volonté objectivé dans et par le système juridique, mais une norme juridique fondée sur la volonté de l'interprète dans chacune de ces décisions (interprétatives).
Ce réalisme décisionniste de l'interprétation semble plus à même de rapprocher les deux modes de normativité, juridique et sociale. Si une norme juridique s'identifie par ses effets dans le système juridique certes, mais que ce système juridique est un ensemble d'actes et non de normes, alors s'estompe en effet la distinction entre un mode spécifiquement normatif juridique et un mode spécifiquement normatif social.

Michel Troper développe cette idée, sans assumer ces présupposés, lorsqu'il présente sa notion de «justification » spécifiquement juridique. Pour lui en effet, une décision sera justifiée et considérée comme une norme lorsque l'interprétation qui est faite par le juge qui met en rapport deux énoncés est jugée correcte dans une société donnée. La part d'acceptation sociale de la norme juridique est bien mise en évidence dans cette théorie de la justification.

La présentation que fait Michel Troper de cette notion de « justification» se trouve, pour l'essentiel, dans son article «Système juridique et État ${ }^{11}$. Dans ce texte, Michel Troper critique les notions kelséniennes de «système juridique » et de "norme». Selon lui, la distinction faite par Kelsen entre «système dynamique » et « système statique » «échoue, telle qu'elle est présentée, à cause de la manière dont Kelsen se représente un ordre normatif et une norme ${ }^{12}$.

Pour développer cette théorie de la normativité, Michel Troper prend en effet appui sur la position de Kelsen en la matière mais pour progressivement la critiquer et la dépasser ${ }^{13}$.

Comme souvent en effet, « le système de Michel Troper pousse la logique du maître de Vienne à ses extrêmes limites, jusqu'à la pervertir totalement ${ }^{14}$. Kelsen présente la norme juridique comme étant la signification objective d'un acte de volonté ${ }^{15}$. Et, on le sait, dans sa conception du système dynamique, Kelsen présente la formation d'une nouvelle norme juridique comme notamment issue d'une norme juridique supérieure valide et inscrite dans un ordre juridique. Donc pour Kelsen, selon Michel Troper, «L'ordre n'est alors qu'un ensemble de relations entre significations ${ }^{16}$. De plus, et toujours selon le juriste autrichien, les interprètes authentiques exercent bien une fonction relevant de la volonté lorsqu'ils interprètent les textes du système juridique ${ }^{17}$.

Selon Michel Troper, chez Kelsen «la norme est seulement une signification et elle n'existe comme signification

10. G. Tusseau, «L'urgence en droit constitutionnel », RFDC, numéro spécial, Le temps et le droit constitutionnel, à paraître en 2009.

11. Repris dans Pour une théorie juridique de l'État, Paris, PUF, 1994 (cité dorénavant PTJE), p. 161-176.

12. Ibid., p. 172. Pour une critique de la lecture de Kelsen par M. Troper, à propos des conceptions statique et dynamique du système juridique, cf. S. Papaefthymiou, «Mind the gap », in L'office du juge: part de souveraineté ou puissance nulle?, O. Cayla, M.-F. Renoux-Zagamé (éd.), Paris - MontSaint-Aignan - Bruxelles, LGDJ - Presses universitaires de Rouen - Bruylant, 2002, p. 195. Selon l'auteur, en effet, «ce n'est pas Kelsen, mais M. Troper qui soutient qu'un système juridique est à la fois statique et dynamique. Kelsen ne parle que d'un "principe d'unité statique" et d'un "principe d'unité dynamique", d'un type de système statique et d'un type de système dynamique, et il ne manque pas d'expliciter sa thèse sur l'absence de contenu prédéterminé» (p. 216).

13. «La théorie réaliste de l'interprétation se sert d'une reconstruction de Kelsen en vue d'introduire sa propre conception en tant que correctifs des erreurs imputées à la théorie de l'école de Vienne» (O. Pfersmann, «Contre le néo-réalisme juridique », RFDC, 2002, p. 299).

14. D. de Bechillon, «Réflexions critiques », RRJ, 1995, p. 248. On préférera plutôt le terme de « reformuler » à celui de " pervertir ».

15. Cf. H. Kelsen, Théorie pure du droit (trad. C. Eisenmann), Paris, Dalloz, 1962, p. 7.

16. «Système juridique et État », PTJE, p. 172-173.

17. Le problème de l'interprétation est envisagé par Kelsen au dernier chapitre de la Théorie pure du droit. Pour une critique de la position de M. Troper qui voit dans le juriste viennois un promoteur de l'idée que l'interprétation n'est pas un acte de connaissance mais uniquement de volonté, cf. O. Pfersmann, «Contre le néo-réalisme juridique», p. 301. L'auteur propose de nommer «acte de concrétisation » ce que Kelsen nomme maladroitement, selon lui, «interprétation authentique». 
qu'en raison et par son insertion dans un ordre, c'est-àdire par sa relation avec une autre norme ${ }^{18}$. Michel Troper tire alors la conclusion qui s'impose en effet : «l'ordre n'est alors qu'un ensemble de relations entre significations $[\ldots] »$.

Cette conception de la normativité est inacceptable pour le philosophe du droit français, pour la simple raison qu' « interpréter, en effet, c'est déterminer la signification de quelque chose. Par ailleurs, une norme est la signification d'un acte de volonté. On ne saurait donc interpréter une norme, parce qu'on ne peut déterminer la signification d'une signification ${ }^{19}$.

Mais ces significations « ne sont pas significations de quelque chose ${ }^{20}$. Donc l'ordre juridique est conçu par Kelsen comme un ensemble de relations « entre éléments qui n'ont pas d'existence hors de ces relations ${ }^{21}$.

Et pour Michel Troper, cette idée est proprement incompréhensible car « on peut concevoir qu'une chose existe et qu'elle soit modifiée ou qu'elle acquiert une signification, lorsqu'elle est placée en relation avec une autre chose, mais on ne peut pas concevoir une relation entre éléments qui n'acquièrent une existence que par elle. L'existence de la relation ne peut précéder celle des éléments, parce qu'on ne peut pas concevoir une relation entre rien et rien ${ }^{22}$.

Ce que veut dire ici Michel Troper, à travers cette critique de la signification chez Kelsen, c'est que si l'on a affaire à un système de normes valides, l'idée d'une interprétation conçue comme un acte de volonté portant sur une norme qui est déjà un acte de volonté ayant signification objective de norme est assez problématique dans la répétition d'actes de volonté qu'elle retient. De plus, et plus généralement, si l'on conçoit la normativité comme préexistant dans le texte qui sert à une nouvelle interprétation, celle-ci portera donc sur quelque chose de déjà normatif, et il est également assez étrange logiquement de faire naître la normativité de la normativité. Enfin, la conception même du système juridique dynamique s'en trouve affectée, car celui-ci étant un ensemble de relations entre significations, " on peut concevoir qu'une chose existe et qu'elle soit modifiée ou qu'elle acquiert une signification, mais on ne peut pas concevoir une relation entre éléments qui n'acquièrent une existence que par elle $»^{23}$.

Ces actes d'interprétation portent sur les textes, c'està-dire des énoncés ne disposant pas encore d'une quelconque valeur normative. Ce qui va faire naître la normativité, c'est l'interprétation et la mise en relation d'énoncés par un interprète authentique du système juridique, par exemple le Conseil constitutionnel ${ }^{24}$.

Le texte interprété n'est donc pas une norme. Il peut avoir un sens que le juge constitutionnel détermine par une activité de connaissance, mais dont la signification objective de norme restera issue de l'acte de volonté interprétatif.

Selon Michel Troper en effet, chez Kelsen, «la difficulté vient donc essentiellement de la coupure établie [... entre les actes de volonté et leur signification ${ }^{25}$. Michel Troper va alors, tout en gardant la structure du raisonnement kelsénien, faire porter l'accent sur les actes et non plus sur les normes. Il transforme ainsi les relations entre significations normatives en relations entre actes de volonté portant sur des énoncés et non plus sur des normes de façon claire lorsqu'il affirme que « dans le système juridique, un acte humain, par exemple un acte de langage, acquiert la signification objective d'une norme, parce qu'un autre acte, qui présente lui-même cette signification, la lui accorde. Les termes de la relation sont donc non les normes, mais les actes, qui acquièrent, les uns par rapport aux autres, la signification de normes ${ }^{26}$.

La critique de Michel Troper porte ici sur la présentation que fait Kelsen, liée à sa théorie de la hiérarchie des normes, de «l'enchaînement » objectif de l'apparition (ou de la création) des normes juridiques. L'auteur français conteste cette possible existence d'une « cascade normative » objective, et quelque part abstraite, qui est supposée exister indépendamment de toute réinscription de l'apparition de la norme juridique dans un acte (concept relevant de la catégorie du fait). Michel Troper conteste finalement l'idée d'un fonctionnement autonome, objectif et indépendant du système juridique.

Comme le note Otto Pfersmann, en présentant les idées de la théorie réaliste de l'interprétation, "prendre au sérieux la théorie kelsénienne de l'interprétation nous obligerait à renverser la problématique : au lieu de rechercher le critère de la validité d'une norme dans une autre norme, il convient de l'identifier dans ce qui est objectivement la signification d'un acte humain, c'est-à-dire l'interprétation-création d'une norme par un organe d'application ${ }^{27}$.

L'apparition d'une norme passe en effet, pour Michel Troper, par l'existence d'un acte; acte qui va acquérir la signification de norme par son rapport avec un autre acte

18. «Système juridique et État», PTJE, p. 172.

19. «Hans Kelsen et la jurisprudence», PTJE, p. 82. C'est nous qui soulignons.

20. «Système juridique et État», PTJE, p. 173.

21. Ibid.

22. Ibid

23. Ibid.

24. Nous prendrons dans les développements qui vont suivre l'exemple du juge constitutionnel, institution qui nous intéresse particulièrement dans le cadre de cette étude, mais la théorie de l'interprétation de M. Troper, en tant que théorie générale du droit, vise à s'appliquer à tous les interprètes authentiques d'un système juridique.

25. "Système juridique et État», PTJE, p. 173.

26. Ibid. C'est nous qui soulignons.

27. O. Pfersmann, «Contre le néo-réalisme juridique», p. 303. Mais pour O. Pfersmann, M. Troper ne voit pas qu’ il est en effet évident que Kelsen ne vise pas ici la norme, mais son support linguistique, en d'autres termes qu'il utilise négligemment le terme norme non seulement pour la norme au sens strict, mais aussi pour qualifier l'énoncé qui l'exprime, alors qu'en d'autres endroits il insiste sur la différence entre les deux ». 
qui va lui accorder cette signification. Le théoricien français opère ainsi un « rétablissement » de la théorie kelsénienne du système normatif en donnant la priorité non plus à la notion de signification mais à celle d'acte de volonté. En changeant l'accentuation que faisait Kelsen dans la proposition théorique «les normes sont la signification objective d'actes de volonté », le point de vue «tropérien » focalise le regard sur la relation entre les actes et non entre les significations.

Pour qu'une norme juridique existe, il faut en repasser par un acte de volonté, acte qui va acquérir la signification de norme par son lien avec un autre acte de volonté qui, lui-même, va ainsi recevoir la signification de norme. Autrement dit, le juge constitutionnel, lorsqu'il interprète une disposition de la Constitution, fait de celle-ci une norme par son acte de volonté à chaque fois renouvelé dans chacune de ses décisions.

On atteint ici le cœur de la théorie réaliste de Michel Troper. Ce changement d'accentuation de la signification objective vers l'acte de volonté du juge opère une véritable révolution du point de vue théorique sur les notions de norme et de système normatif.

En effet, si Michel Troper met l'accent sur l'acte de volonté, comme processus ${ }^{28}$ et comme $~ "$ fait ${ }^{29}$, il lui faut alors rebâtir une théorie qui puisse aboutir à donner la qualification de norme juridique à l'expression de ces actes de volonté juridictionnels. La construction de cette théorie passe par le développement du concept de justification.

Michel Troper veut présenter, c'est le titre de son ouvrage, une théorie juridique de l'État, et donc, selon l'identité kelsénienne bien connue qu'il conserve, du droit. Il lui faut donc découvrir un moyen de faire des actes de volonté des interprètes et donc du juge constitutionnel, des objets juridiques. Mais ceci sans l'appui d'une quelconque notion de signification « objective » indépendante de l'existence de normes déjà existantes et chargées de fonder la validité de la norme à venir. C'est bien l'autorité capable d' « agir» le droit qui devient le cœur de la théorie tropérienne de la normativité ${ }^{30}$.

Cette mise en avant de l'auteur de l'acte, de l'énoncé (appellation neutre du texte qui va bientôt devenir norme) est conforme au présupposé de la théorie réaliste du droit qui veut que " the law is what judge made law ${ }^{31}$. Le droit, et avec lui les catégories conceptuelles de normativité, de juridicité, est ainsi présenté comme ne pouvant être perçu théoriquement qu'à travers le point de vue de l'autorité qui agit le droit, et donc principalement à travers l'activité juridictionnelle. Toute autorité de ce type produit un discours, et ce discours, par la forme qu'il prend, va faire apparaître les normes. Selon Michel Troper, «Il est possible d'intégrer à la présentation du système normatif une théorie de l'interprétation comprise comme activité volontaire de détermination d'une signification. Un énoncé aura la signification d'une norme, si son auteur le met en relation avec un énoncé, qu'il interprète lui-même. Comme la signification d'un énoncé est une norme, la justification apparaît alors comme la mise en relation de deux énoncés dont les significations sont déterminées par une même autorité. Les termes de la relation ne sont pas des normes, mais des actes, qui reçoivent la signification de normes, l'un par l'activité d'interprétation, le second par sa mise en relation avec le premier une fois que cette activité a été accomplie $»^{32}$.

Cet extrait nous permet de définir la justification chez Michel Troper comme étant la mise en relation de deux énoncés dont les significations sont déterminées par la même autorité. Toute l'alchimie normative présentée par le théoricien français a pour origine et pour scène un auteur unique. Si l'on prend comme exemple le Conseil constitutionnel, dans le schéma réaliste, celui-ci interprète le texte constitutionnel et lui donne ainsi la signification de norme. Puis il met cette norme constitutionnelle en relation avec un autre acte, par exemple une loi, et cette opération de liaison donne ainsi la signification de norme à ce second acte. Cette opération de liaison, Michel Troper l'appelle «justification».

C'est donc bien la même autorité qui produit la normativité et qui la justifie en produisant une autre normativité qui se trouve elle-même justifiée par cette opération. Mais tout ceci se produit du point de vue de l'autorité créatrice. L'apparition de la normativité constitutionnelle et législative, dans une décision du Conseil constitutionnel, se fait donc uniquement relativement à cette décision et par l'acte de volonté du Conseil.

On pourrait alors objecter à Michel Troper que si l'on ne prend que ce point de vue interne à la décision juridictionnelle, il n'est alors pas possible de passer de l'acte de l'autorité en question, perçu comme un fait, à son appréhension comme phénomène juridique. Michel Troper créerait, si l'on peut dire, une nomade sans porte ni fenêtres, et cette activité du juge ne serait pas visible de l'extérieur, c'est-à-dire pas théorisable comme étant un processus de création du droit. Ainsi le caractère juridique de la norme viendrait uniquement de cette activité du juge constitutionnel, ce qui rendrait impossible la qualification de cet évènement d'objet spécifiquement juridique. Par cette reformulation de la notion kelsénienne de la signification, Michel Troper serait, selon Gérard Timsit, « au bout du compte, pris dans l'engrenage de ce mécanisme catastrophique de transmutation de tout le droit en fait ${ }^{33}$.

28. Chez Michel Troper en effet, le processus d'apparition de la normativité l'emporte sur la norme elle-même comme objet de la science du droit, «ce qui est objet de connaissance n'est jamais la signification elle-même, mais seulement l'attribution de la signification " (M. Troper, «Réplique à Otto Pfersmann ", RFDC, 2002, p. 347 ; c'est nous qui soulignons).

29. L'acte d'attribution de la signification, c'est-à-dire l'interprétation authentique, relève bien du domaine du fait que la science du droit doit décrire, et cette " qualité de l'interprétation est d'ailleurs une question de pur fait» (ibid., p. 343).

30. «La relation n'est pas elle-même objective, mais seulement une mise en relation par l'auteur d'un énoncé » («Système juridique et État », PTJE, p. 174).

31. Cité par M. Troper, «Le problème de l'interprétation et la théorie de la supralégalité constitutionnelle», PTJE, p. 313.

32. «Système juridique et État», PTJE, p. 174.

33. G. Timsit, Les figures du jugement, Paris, PUF, 1993, p. 119. Selon l'auteur, ce résultat «n'est guère rassurant». Même si, pour lui, «Michel Troper a eu le souci, avec cette analyse, de mieux tenir compte de la place des faits dans le droit» (p. 116), la conception de ce dernier empêche de parler de droit. 
Mais la méthode de Michel Troper échappe pour partie à cette critique. Celui-ci a en effet pris soin, avant de décrire "l'expérience normative intérieure», d'ouvrir une porte sur un regard extérieur, regard extérieur qui va venir « justifier» l'ensemble de cette activité intérieure.

Et ce regard, ce point de vue extérieur, Michel Troper le qualifie également d'opération de justification.

Cette autre occurrence du terme de "justification » dans le même article, Michel Troper la présente ainsi : «Le caractère obligatoire ou validité n'est jamais une propriété objective de la norme, ni son mode d'existence. La relation n'est pas elle-même objective, mais seulement une mise en relation par l'auteur d'un énoncé. Si cette mise en relation est jugée adéquate dans la société considérée, ce qui est une question de fait, la décision est considérée comme justifiée et l'on peut dire qu'elle présente le caractère d'une norme. Le système juridique n'est alors pas autre chose qu'un système de justification. Le problème de l'applicabilité de la logique au droit ne se pose pas, puisque la justification est seulement une question de fait. Une décision justifiée est une décision dont la justification est jugée correcte dans la société considérée. Ainsi, on peut admettre, ce qui n'était pas possible avec la présentation de Kelsen, que, dans un système statique, un énoncé acquiert la signification de norme lorsque son auteur affirme, sans que cela soit contesté, que son contenu est déduit du contenu d'un autre énoncé ${ }^{34}$.

Michel Troper nous présente bien là un autre emploi du terme de «justification». Cette seconde justification, ou «justification sociale», a pour fonction de faire une norme de la décision d'une autorité. Et ce processus normatif est, selon Michel Troper, une simple question de fait, en ce qu'il consiste en une reconnaissance par la société du caractère adéquat (correct) de l'opération, décrite cidessus, d'interprétation-justification intérieure.

Cette justification sociale est l'expression du jugement que porte la société destinataire de cette décision sur celleci. Et ce jugement, s'il est «positif ${ }^{35}$, confère à son tour le caractère de norme au texte de la décision lui-même, c'est-à-dire qu'il justifie que cette décision produise certains effets. On peut donc, en un certain sens, dire que la société justifie la justification opérée par l'autorité auteur du texte de la décision.

Ainsi lorsque le Conseil constitutionnel décide de la conformité ou au contraire de la contrariété d'une disposition législative ou conventionnelle à la Constitution, sa décision produit-elle la normativité de ces deux textes, mais elle est également justifiée par l'acceptation qu'elle reçoit de la part de la société, c'est-à-dire notamment des autres acteurs du système juridique, mais aussi plus largement de l'ensemble des citoyens.

Il s'agit donc bien d'une justification de justification à laquelle on assiste de la part de la société en question. Le système juridique, qui est notamment l'ensemble des décisions des autorités juridiques, peut donc être conçu comme un système de justification sociale portant sur des justifications opérées par les autorités habilitées à dire le droit $^{36}$. Le système juridique compris comme ensemble des normes contient donc bien les normes créées par l'activité des autorités juridiques pour arriver à une décision (justification intérieure) et les normes résultats de cette activité créatrice, c'est-à-dire les décisions elles-mêmes, justifiées dans la société considérée.

La théorie des normes de Michel Troper consiste bien en un système dans lequel on constate la coexistence de deux types de justifications normatives différentes. Mais alors ceci nous rappelle peut être que ce qui est exceptionnel est en fait ce qu'une société donnée, à un moment donné, reconnaît comme exceptionnel, c'est-à-dire refuse de reconnaître comme une norme justifiée en le maintenant hors de la juridicité.

Mais force est de reconnaitre que cette conception de la normativité largement fondée sur un jugement social permet de rapidement absorber l'exceptionnel dans la norme et permet difficilement de faire la part de ce qui relève spécifiquement de la signification juridique, de la règle de droit, et ce qui relève de sa mise en œuvre.

Ce point, qui nous suit depuis le début de ces lignes, peut maintenant être abordé sous un autre angle qui va peut-être permettre de rapprocher plus directement ce questionnement théorique et la problématique des droits fondamentaux.

\section{Juger de l'action sur la réalité par la norme. L'exemple de l'égalité et de ses effets}

L'égalité est l'un des principes juridiques les plus importants dans un État de droit. En en faisant la matrice, le principe régulateur du système des droits (fondamentaux), on espère que cette égalité des droits affirmée dans une norme juridique aura des effets dans les faits, c'est-àdire que de situations égales juridiquement sortiront des situations égales dans les faits. Cet espoir légitime d'une égalité dans les faits fondée et garantie par le droit pose une question de théorie du droit importante, essentielle, à savoir celle du rapport entre l'affirmation d'un principe sous une forme juridique et sa concrétisation factuelle.

Il est légitime d'estimer que si sont élaborées des normes juridiques en matière de droits fondamentaux sous le respect du principe d'égalité, c'est dans l'espoir que dans la réalité - que l'on appellera par commodité factuelle ou "sociale»-les individus soient effectivement plus égaux. Mais ce lien entre la signification d'un principe juridique et son effectivité hors de sa stricte formulation juridique est d'une grande difficulté à cerner et à formuler.

34. "Système juridique et État », PTJE, p. 174. C’est nous qui soulignons.

35. Il faut noter que M. Troper ne nous présente pas comment se manifeste, s'exprime, cette approbation ou cette réprobation sociale.

36. On se permettra donc de réécrire la formulation de M. Troper : «Le système juridique n'est alors pas autre chose qu'un système de justificationS » (c'est nous qui ajoutons). 
L'interrogation peut prendre une formulation de ce genre : comment juge-t-on tout d'abord de l'égalité de situations juridiques, et comment peut-on juger ensuite de la connexion entre cette égalité juridique et une situation de fait (rendue par là) égalitaire?

Commençons par planter un décor philosophique général avant de revenir sur le principe d'égalité proprement dit.

Partons de quelques pages de l'indispensable ouvrage de Jacques Bouveresse La force de la règle ${ }^{37}$, et tout d'abord de celles consacrées à ce que l'on nomme en philosophie du langage «l'idée du corps de signification».

Les réflexions du philosophe français visent en premier lieu dans ces pages, en développant les conceptions de Wittgenstein, à se débarrasser de l'idée que la signification de chacun des termes d'une proposition nécessaire permettrait à elle seule de déterminer la signification de la proposition elle-même dans son ensemble et surtout la signification des propositions qui en sont déduites, qui se présentent comme son application, sa mise en œuvre.

Par proposition nécessaire on entend des propositions qui expriment ce qui se présente comme une nécessité, par opposition à ce qui n'est que contingent, accidentel, dépendant d'une certaine situation de fait. Les énoncés mathématiques sont un type de proposition nécessaire, mais il en existe d'autres. Une manière d'identifier un énoncé, une proposition nécessaire est de se demander si ce sur quoi porte la proposition est invariablement vrai, vrai a priori, c'est-à-dire donc nécessairement vrai.

Pour le dire autrement alors, la critique de Wittgenstein porte sur l'idée, qui paraît en effet assez triviale et de bon sens, qu'un terme, un signe, lorsqu'il est utilisé dans une proposition, reçoit par là une signification invariante qu'il transporte ensuite avec lui, que l'assemblage des significations des termes d'une phrase dans le respect des règles de la syntaxe permet de donner à cette phrase une signification globale et que, dans le cas où une telle proposition appellerait une application (si c'est donc une règle), alors cette signification de la proposition de départ déterminerait a priori complètement celle d'arrivée (l'application).

Cette critique de la conception de la façon dont signifient les énoncés d'un certain type (les énoncés nécessaires ou « grammaticaux », comme le dit Wittgenstein) aboutit en effet selon Jacques Bouveresse également à une critique d'une conception qui lui est liée, celle du « corps de la règle », conception qui consiste à envisager l'énoncé d'une règle « comme un dépôt à partir duquel se déroule l'usage ou une machine logique qui engendre des applications de son propre chef ${ }^{38}$.

Cette critique d'une telle conception de l'application automatique de la règle au cas, de la proposition nécessaire à son application ou à sa mise en œuvre, est assez contre-intuitive relativement aux conceptions les plus répandues en philosophie du langage. Elle apparaît plus naturelle en revanche au sens commun du juriste, si l'on passe, avec toutes les précautions nécessaires bien sûr (en évitant les vertiges de l'analogie), de son étude dans le cadre philosophique et à propos des énoncés nécessaires, à son usage (sans jeu de mot) dans le cadre de la mise en œuvre des « règles » juridiques.

Mais s’il faut évidemment distinguer les énoncés nécessaires dans le champ par exemple des mathématiques des énoncés que l'on appelle « règles » ou normes dans le cadre juridique, des rapprochements intéressants peuvent quand même apparaître qui invitent à se poser des questions sur ce en quoi consistent certaines règles juridiques comme le principe d'égalité.

La Déclaration de 1789, intégrée au bloc de constitutionnalité français, prévoit dans son article $1^{\mathrm{er}}$ on le sait que «Les hommes naissent et demeurent libres et égaux en droits » et que «Les distinctions sociales ne peuvent être fondées que sur l'utilité commune ». L'article 6 du même texte précise que la loi est l'expression de la volonté générale et qu' «elle doit être la même pour tous, soit qu'elle protège, soit qu'elle punisse».

La réflexion autour du principe d'égalité tourne souvent autour de l'idée que l'égalité en droits serait un moyen (indispensable) de l'égalité en fait. C'est pour avoir une effective égalité de fait que l'on prévoirait dans une règle de droit l'égalité des droits des êtres humains.

Et la formulation de l'article $1^{\text {er }}$ de la Déclaration de 1789 prête tout particulièrement à la recherche de ce lien entre le droit et les faits, car la seconde phrase nous apprend que des distinctions sociales peuvent être fondées sur l'utilité commune et autoriser des aménagements à la formule très générale de la première phrase qui pour sa part ne vise que l'égalité juridique des droits.

Cette idée d'un lien entre l'égalité juridique et l'égalité des droits apparaît clairement dans le débat sur les discriminations positives, a contrario il est vrai : c'est pour rétablir l'égalité de fait que l'on rompt, que l'on déroge à l'égalité des droits. On introduit la norme, dans un aspect dérogatoire, pour rétablir dans les faits une situation exceptionnelle.

On part d'un constat empirique, sociologique, comme celui par exemple que les personnes qui habitent des «zones urbaines sensibles » réussissent statistiquement moins à accéder à certains emplois publics pour déroger juridiquement à l'application «classique », "normale» du principe d'égalité, qui dans sa version juridique ne permet pas de distinguer, dans certains contextes, entre les citoyens en fonction de leurs origines géographiques (sans parler de leur origine ethnique bien sûr). C’est pour une utilité commune que l'on fait une distinction juridique. Mais quel intérêt d'en avoir appelé à la philosophie du langage pour parler à nouveau de ce problème largement déjà discuté?

Tout d'abord on peut se demander en quoi l'article de la Déclaration contient une règle, et quel type de règle ou de norme. Difficile de voir le principe d'égalité, même dans une formulation à l'indicatif, comme une norme de type uniquement descriptive d'un certain état de fait. En effet il apparaît assez clairement que cet énoncé, cet article

37. J. Bouveresse, La force de la règle, Paris, Éditions de Minuit, 1987.

38. Ibid., p. 33, citant G.P. Baker, P.M.S. Hacker, Skepticism, Rules and Language, Oxford, Blackwell, 1984, p. 17. 
de la Déclaration ne décrit pas ce qu'il en est en fait de l'égalité, mais a comme fonction de pouvoir parler de l'égalité non plus comme un fait mais comme quelque chose de juridique, quelque chose qui « doit être», une prescription, un commandement, une injonction, un ordre. Même sans revenir à l'infini débat sur la nature de la règle ou de la norme juridique, on peut dire qu'elle n'est pas une proposition descriptive d'un état de fait à un moment donné au même sens où un énoncé comme « Les hommes vivent en moyenne en France jusqu'à 74 ans et trois mois » est la description d'un tel état de fait (proposition qui implique qu'existent des normes de description qui permettent de formuler ce jugement).

On pourrait aussi voir le principe d'égalité comme une règle du type «Tous les hommes doivent être dans une situation égale en matière de protection de leurs droits (ou doivent être traités de façon égale, identique en matière d'accès à leurs droits) et cela leur provoquera alors une situation de fait égale, égalitaire ", et alors il apparaît comme une règle semblable dans sa formulation à un énoncé du genre "Tout corps plongé dans l'eau reçoit une poussée verticale égale au poids du volume d'eau déplacé ». La règle juridique est alors vue comme une loi physique qui permet de décrire un certain état de la réalité " physique» et permet de faire certaines prédictions factuelles (si je plonge un tel corps dans l'eau alors telle poussée sera exercée...). Il faut alors également admettre que cette règle est tirée, inférée de la réalité (il faut alors dire selon quelle modalité, et quelle est la «loi» qu'elle établit).

On pourrait également essayer de voir la règle juridique comme un énoncé nécessaire, une proposition grammaticale comme le dit Wittgenstein, c'est-à-dire une proposition qui a pour objet non pas de décrire la réalité ou d'établir une loi physique mais de permettre de donner une signification à des propositions hypothético-prédictives ou empiriques sur la réalité.

Une difficulté réside dans le fait que, comme le dit Jacques Bouveresse, un des traits de la proposition nécessaire ou grammaticale est de ne correspondre à rien dans la réalité, au sens où elle ne doit pas sa normativité à un rapport avec la réalité, avec certains faits, même d'un type particulier (des « faits nécessaires » dont elle serait la description).

C'est la réalité qui au contraire peut seule avoir une signification à travers les propositions grammaticales et les applications qui en sont faites ${ }^{39}$.

Et de ceci découle quelque chose d'intéressant: un jugement d'identité d'une situation ne vient pas de l'identité naturelle de la situation, il ne peut venir que de l'usage, du choix de l'usage d'une règle qui a pour conséquence, par sa mise en œuvre, de nous faire dire qu'il y a identité de cas.
«C'est en fait uniquement la règle elle-même (ou plus exactement, ce que nous considérons comme la façon correcte de l'appliquer) qui donne son sens à ce que l'on appelle, en l'occurrence, "faire la même chose". C'est la règle qui crée le point de vue auquel tous ces cas, aussi différents que possible à d'autres égards, peuvent être identifiés et perçus comme ayant été traités de façon identique ${ }^{40}$.

Revenons au principe d'égalité et posons maintenant la question clairement : comment peut-on dire que deux hommes sont égaux en droits, au sens de la Déclaration de 1789 ? Le plus simple est de dire qu'ils ont les mêmes droits, ou plutôt que dans une situation identique, ils ont droit aux mêmes droits, au même traitement juridique. C'est ce qu'exprime le Conseil constitutionnel dans son «considérant type » sur le principe d'égalité: «le principe d'égalité ne s'oppose ni à ce que le législateur règle de façon différente des situations différentes, ni à ce qu'il déroge à l'égalité pour des raisons d'intérêt général, pourvu que, dans l'un et l'autre cas, la différence de traitement qui en résulte soit en rapport avec l'objet de la loi qui l'établit » ${ }^{41}$.

Le problème vient de l'expression «situation identique» bien sûr (et du terme «même» qui l'accompagne). Si l'on dit qu'il s'agit d'une situation juridique identique, on se retrouve dans un cas assez étonnant car l'énoncé sur l'égalité devient alors un énoncé descriptif, qui sert à dire que deux hommes sont dans la même situation juridique, c'est-à-dire qu'ils ont les mêmes droits, que le système juridique leur reconnaît les mêmes droits, qu'ils ont un statut juridique identique (ou équivalent, le débat ne porte pas ici sur la notion d'égalité comme identité ou comme similarité ou équivalence).

S'il s'agit d'une situation de fait (si l'on veut toujours rapprocher égalité de droits et égalité en fait), alors il faut voir comment l'égalité juridique provoque des situations de fait identiques (ou similaires, ou équivalente). Mais le jugement d'identité ne peut venir que de la mise en œuvre d'une règle qui nous permet de dire qu'il y a bien identité, car comme on l'a vu pour dire qu'on a affaire à « la même situation ", il faut une règle valide $a$ priori qui nous permet de nous prononcer sur la conformité du cas avec la règle et c'est de cette même règle appliquée correctement à deux cas que naît l'identité (la similarité ou l'équivalence).

Mais alors il faut admettre que l'identité de fait, dans les faits, vient de la correcte application à deux cas de la règle juridique, c'est-à-dire de ce que nous appelons la correcte application à deux cas de la même règle. L'identité de fait n'est pas alors le produit d'une règle non juridique, de type sociologique par exemple.

Et alors surgit un problème: comment alors savoir jamais si l'on est dans une situation de fait identique qui appelle l'application du principe d'égalité juridique, ou inversement dit, comment parler des effets dans la réalité

39. Cf. notamment le chapitre sur «L'autonomie de la grammaire », p. 21 et celui sur "À quoi correspond une règle », p. 40.

40. J. Bouveresse, La force de la règle, p. 38 .

41. Par exemple dans la décision 2006-540 DC du 27 juillet 2006. Le conseil précise, dans une décision postérieure, que "si, en règle générale, le principe d'égalité impose de traiter de la même façon des personnes qui se trouvent dans la même situation, il n'en résulte pas pour autant qu'il oblige à traiter différemment des personnes se trouvant dans des situations différentes », 2006-541 DC du 28 septembre 2006. 
(au sens social) de la règle de droit relative au principe d'égalité (c'est-à-dire comment sait-on que c'est bien à deux situations identiques que l'on va (doit?) appliquer le principe d'égalité)?

Comment fait-on le lien entre la règle juridique et ses applications juridiques et un cas de situation factuelle, que l'on voudrait identique, si c'est bien l'égalité dans les faits (ou une "plus grande égalité») qui est recherchée par le droit?

Dans notre optique, si l'on soutient que seule ce que l'on nomme une correcte application d'une même règle (une opération de jugement) permet l'identification (ou est constitutive) d'une identité, il faudrait donc trouver une autre façon de décrire la réalité à partir de propositions a priori, spécifiquement factuelles ou sociales (par opposition à juridique), mais qui peuvent être reliées à nos propositions juridiques, sinon on perd l'idée que la norme juridique a des effets dans la réalité en ce qui concerne cette égalité factuelle, sociale.

Comment peuvent donc communiquer entre elles ces propositions juridiques et factuelles, ou « sociales » ?

On peut dans un premier temps être déstabilisé car la réponse la plus logique est qu'elles ne peuvent pas communiquer, si on veut dire par là que l'on pourrait en effet assimiler égalité juridique et égalité sociale, ou si l'on voulait dire que la réalité pourrait trancher d'elle-même un conflit par exemple sur l'application à faire du principe d'égalité dans un cas particulier. Il n'y a pas d'égalité naturelle, de fait naturel de l'égalité en ce sens-là.

Mais comme Wittgenstein y insiste souvent, et comme le rappelle Jacques Bouveresse, dire que les règles de grammaire ou les propositions nécessaires sont autonomes par rapport à la réalité ne veut pas dire que le langage des règles est autoréférentiel et que le langage ne se réfere pas à quelque chose d'extérieur à lui, qu'il n'intervient pas dans la réalité ${ }^{42}$.

Mais qu'entend-on réellement par cet appel à la « réalité »? Si l'on cherche un espace «neutre» hors langage, d'où l'on pourrait dire ce qu'il est correct de dire en appliquant des règles, ou ce qu'il en est du rapport entre les règles et la "réalité», alors sans doute aucune réalité de ce genre n'existe-t-elle. Et c'est justement tout le travail de Wittgenstein de montrer que « ce qui semble être une correspondance métaphysique est en fait une articulation intragrammaticale ${ }^{43}$.

Mais cela ne veut pas dire qu'il n'y a pas d'égalité en fait, ou dans les faits. Cela veut seulement dire qu'on ne peut pas la découvrir dans des faits d'une nature particulière, dans l'invocation d'une réalité particulière qui pourrait venir dire, indépendamment d'un certain état de fait (si on peut dire...) ou plutôt d'un certain état des faits dont on juge à travers des règles qui ne sont pas que juridiques, si l'on est réellement dans une situation d'égalité.

Il faudrait beaucoup de développements encore pour voir si les règles juridiques, et surtout, ce qui est toujours le problème, si toutes les règles juridiques peuvent être réellement appréhendées comme des règles grammaticales (d'un genre spécifique, car à la différence de ce que Wittgenstein soutient pour les propositions mathématiques, les propositions juridiques comme celles sur l'égalité ont sans doute un certain contenu cognitif), comme des propositions signifiantes a priori, et pour approfondir encore le lien avec la réalité, qui prend la voie d'un lien avec l'action justifiée en référence à une règle juridique ${ }^{44}$, mais on peut voir à travers cette réflexion sur la règle comment l'égalité de fait qui est poursuivie à travers l'égalité juridique entretient avec cette dernière des liens complexes qui en tout cas semblent nous impliquer d'une façon particulière dans la décision, dans le choix d'appliquer certaines règles, qui ne peuvent être uniquement juridiques et qui seules nous permettent d'en parler et qui guident notre action en lui donnant un sens et une justification.

La dernière remarque pourrait alors être pour se demander si ce n'est pas cette césure entre le droit et les faits, que nous révélait particulièrement la distinction entre la norme et l'exception, entre un domaine qui serait propre à la normativité juridique et un domaine « factuel », qui rend difficile de penser ces liens. Comme le notait Wittgenstein dans ses Carnets, «n'y a-t-il aucun domaine en dehors des faits?».

42. Cf. le chapitre "Conceptualisme et réalisme», dans La force de la règle, p. 57 sq.

43. G.P. Baker, P.M.S. Hacker, Skepticism, Rules and Language, cité par J. Bouveresse, La force de la règle, p. 59.

44. Cf. pour cela les développements de Vincent Descombes sur la nécessité pratique dans son ouvrage Le raisonnement de l’Ours, Paris, Seuil 2007, not. p. 435 sq. 